\title{
Phononic Crystals With Archimedean-like Tiling: Band Structure and the Transformation of Sound
}

\section{Zafer Ozer, Amirullah M. Mamedov \& Ekmel Ozbay}

To cite this article: Zafer Ozer, Amirullah M. Mamedov \& Ekmel Ozbay (2021) Phononic Crystals With Archimedean-like Tiling: Band Structure and the Transformation of Sound, Integrated Ferroelectrics, 220:1, 132-139, DOI: 10.1080/10584587.2021.1921542

To link to this article: https://doi.org/10.1080/10584587.2021.1921542

Published online: 01 Dec 2021.

Submit your article to this journal

IIl Article views: 26

Q View related articles

View Crossmark data [ 


\title{
Phononic Crystals With Archimedean-like Tiling: Band Structure and the Transformation of Sound
}

\author{
Zafer Ozer ${ }^{a}$, Amirullah M. Mamedov ${ }^{\mathrm{b}, \mathrm{c}}$, and Ekmel Ozbay ${ }^{\mathrm{b}}$ \\ ${ }^{a}$ Mersin Vocational High School, Mersin University, Mersin, Turkey; ${ }^{b}$ Nanotechnology Research Center, \\ Bilkent University, Ankara, Turkey; ' International Scientific Center, Baku State University, \\ Baku, Azerbaijan
}

\begin{abstract}
In this study, we investigate acoustic wave propagation in 3D phononic crystal (PnC) slabs wherein scatterers have different cross sections by using the finite-element method. The PnC consists of scatterers embedded in the host material arranged in a square lattice and honeycomb lattice in different patterns (bathroom and ladybug tiling in Archimedean-like tiling). By determining the eigenmodes and band gaps, complete and accurate band structures and transmission spectra are obtained. Compared to traditional square lattice $\mathrm{PnCs}$, it has been observed that the bands obtained in pattern structures may have some advantages in terms of width and position. It was also shown that the low-frequency response of two Archimedean-like structures was similar with respect to the traditional square lattice.
\end{abstract}

\section{ARTICLE HISTORY}

Received 23 November 2020

Accepted 25 March 2021

\section{KEYWORDS}

Archimedean-like tiling; phononic crystal; finiteelement method

\section{Introduction}

Phononic crystals $(\mathrm{PnC})$, the acoustic wave analogy of the photonic crystals, are periodic structures consisting of the inclusions in a homogeneous host material with forbidden bands where some frequencies are completely reflected. PnC can be used for manipulating acoustic and elastic waves.

There are many interesting studies in PnCs that have been both numerically and experimentally studied including a lot of already tested applications (waveguides, resonators, filters, or photonic integrated circuits) that have emerged nowadays [2-6]. In spite of Archimedean lattices having been known since ancient times, the first were elucidated and reported by Kepler. Because these structures are symmetrical, both the unit cell and a related Brillouin region can be defined, and the band structure can be obtained by the finite-element method (FEM). The symmetry of these patterns is similar to the squares, triangles, and honeycomb cages that have been used so far in the investigation of photonic crystals. Archimedes-like structures may have some advantages over traditional lattice PnCs in terms of full band gaps as well as their width and location. In addition, the same size Archimedes-like structure has similar wave responses at lower frequencies as traditional square lattice. Due to the wide band structure, these structures 


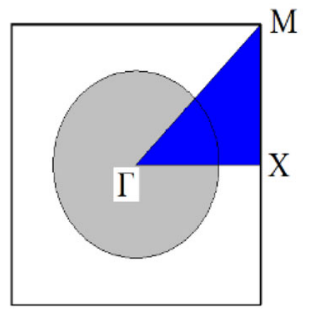

a)

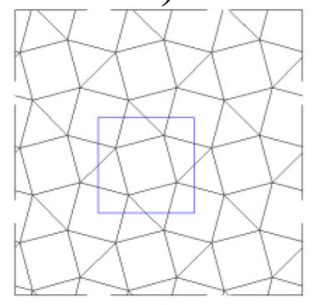

c)

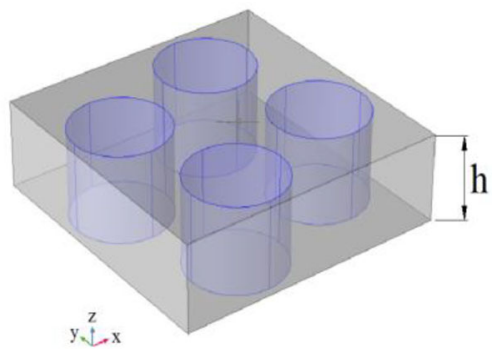

b)

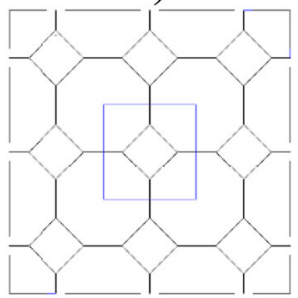

d)

Figure 1. (a) Traditional square lattice unit cell (blue square) b) 3D unit cell c) Archimedean-like tilling's in Grünbaum and Shephard notation $\left(3^{2}, 4,3,4\right)$ (ladybug) and d) $\left(4,8^{2}\right)$ (bathroom).

attract the attention of researchers regarding both phononic and photonic crystals [7-9].

In the present study, different patterned square lattice and honeycomb lattice PnC with different cross-sections scatterers in an epoxy matrix were investigated based on (FEM). PnCs were first identified as square lattice unit cells and honeycomb lattice with the band structure obtained in the direction of the $M-\Gamma-\mathrm{X}-\mathrm{M}$ and $\Gamma-\mathrm{M}-\mathrm{K}-\Gamma$. Figure 1a shows the unit cells of traditional PnC and Figure $1 \mathrm{~b}$ shows a three-dimensional (3 D) unit cell for Grünbaum and Shephard notation $\left(3^{2}, 4,3,4\right)$.

\section{Method}

The elastic wave in an inhomogeneous and anisotropic solid can be expressed by the Equation (1).

$$
-\nabla[C(r): \nabla u(r)]=\omega^{2} \cdot \rho(r) \cdot u(r)
$$

where, $r=(x, y, z)$ is position vector, $\rho(r)$ is mass density, $\omega$ is angular frequency and $C(r)$ elastic tensor, respectively. Due to periodicity, elastic displacement can be defined by Bloch's theorem.

$$
u(r)=u_{k}(r) \cdot e^{i(k r)}
$$

If two-dimensional (2 D) PnC consisting of periodically arranged cylinders in a host material is assumed to be infinite, the periodic structure can be represented by appropriate unit cells as shown in Figures 1 a and $2 \mathrm{a}$.

In order to obtain the band structure of $\mathrm{PnC}$, the Floquet periodicity conditions were applied to the sides of each unit cell. The FEM software is then used to solve the eigenvalue Equation (1) above to obtain mode frequencies. 


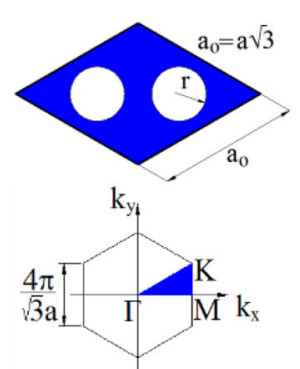

a)

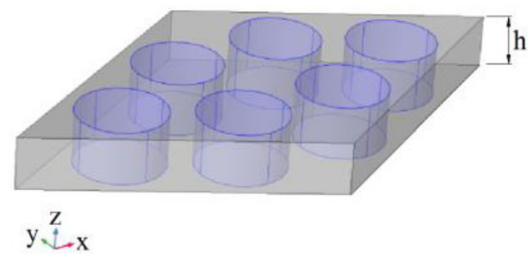

b)

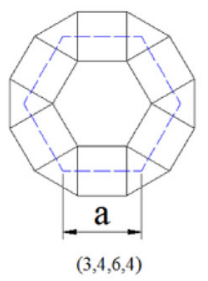

c)

Figure 2. (a) Honeycomb lattice and reciprocal lattice, (b) 3D unit cell, Archimedean tilling in honeycomb lattice in Grünbaum and Shephard notation (c) $(3,4,6,4)$.

A phononic band diagram of a $3 \mathrm{D}$ unit cell of the $\mathrm{PnC} \omega=\omega(k)$ with dimensionless frequencies $\left(\omega a / 2 \pi c_{s_{-} \text {epoxy }}\right)$ were plotted vs. the wavevector $k$ along the $(\mathrm{M}-\Gamma-\mathrm{X}-\mathrm{M})$ direction for square lattice and along the $(\Gamma-\mathrm{M}-\mathrm{K}-\Gamma)$ direction for honeycomb lattice in the square Brillouin zone, respectively.

The PnC consist of various materials included in the epoxy matrix with a different cross section. Figure 1 shows the traditional square lattice unit cell Archimedean-like tilling in Grünbaum and Shephard notation $\left(3^{2}, 4,3,4\right)$ (ladybug) (Figure $1 \mathrm{~b}$ and c) $\left(4,8^{2}\right)$ (bathroom) $\mathrm{PnC}$ in the epoxy matrix with square lattice (Figure 1c). The filling factor set to $f=0.4$ in all unit cells and the high of unit cells are taken $h=0.3^{*} a$.

Figure 2 shows Archimedean-like tilling in form $(3,4,6,4)$. Density, Young's modulus, Poisson ratio, pressure-wave speed, and shear-wave speed of epoxy matrix are $\rho=1180 \mathrm{~kg} / \mathrm{m}^{3}$, Young's modulus $E=3.85 \times 10^{9} \mathrm{~Pa}, \nu=0.37, c_{\mathrm{p}}=2535 \mathrm{~m} / \mathrm{s}$, and shearwave speed $c_{\mathrm{s}}=1157 \mathrm{~m} / \mathrm{s}$, respectively, and the mechanical properties of other materials are taken from the literature $[10,11]$.

Figure 3 shows the boundary conditions of the top view of $3 \mathrm{D}$ finite array used for calculating the transmission. Symmetry boundary conditions (SBC) applied the top and bottom sides, perfectly matched layer (pml) conditions for both ends. In Figure 3, the red line shows the excitation source. For simulating the P, SH, and SV waves, a surface load applied along the $x, y$, and $z$ directions, respectively, for Rayleigh and Love wave, an edge load applied along the $z$ and $y$ directions, respectively. Transmission is calculated by the $20 \cdot \log _{10}\left(u_{B} / u_{A}\right)$ formula.

\section{Discussion}

In this section, a detailed calculation and discussion will be presented for $3 \mathrm{D}$ PnCs with Archimedean-like tiling, "ladybug," and "bathroom." The lattices are composed of 


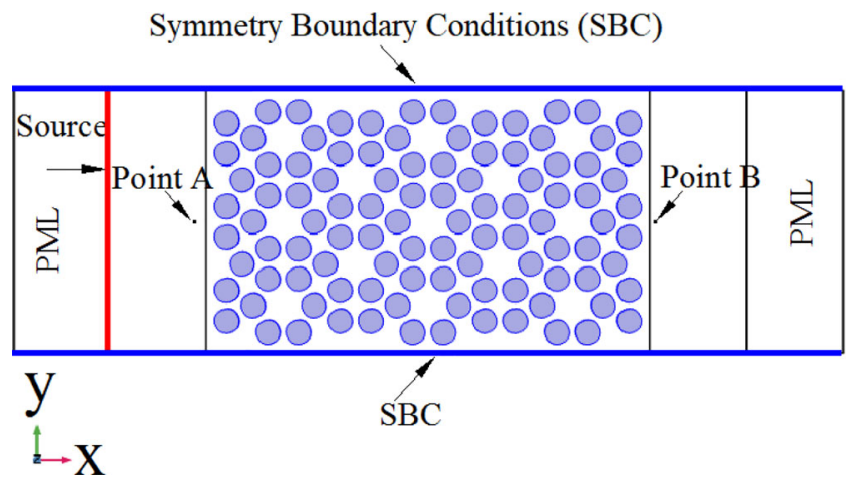

Figure 3. Finite structure and boundary conditions for calculating the transmission for Archimedean tilling in honeycomb lattice.

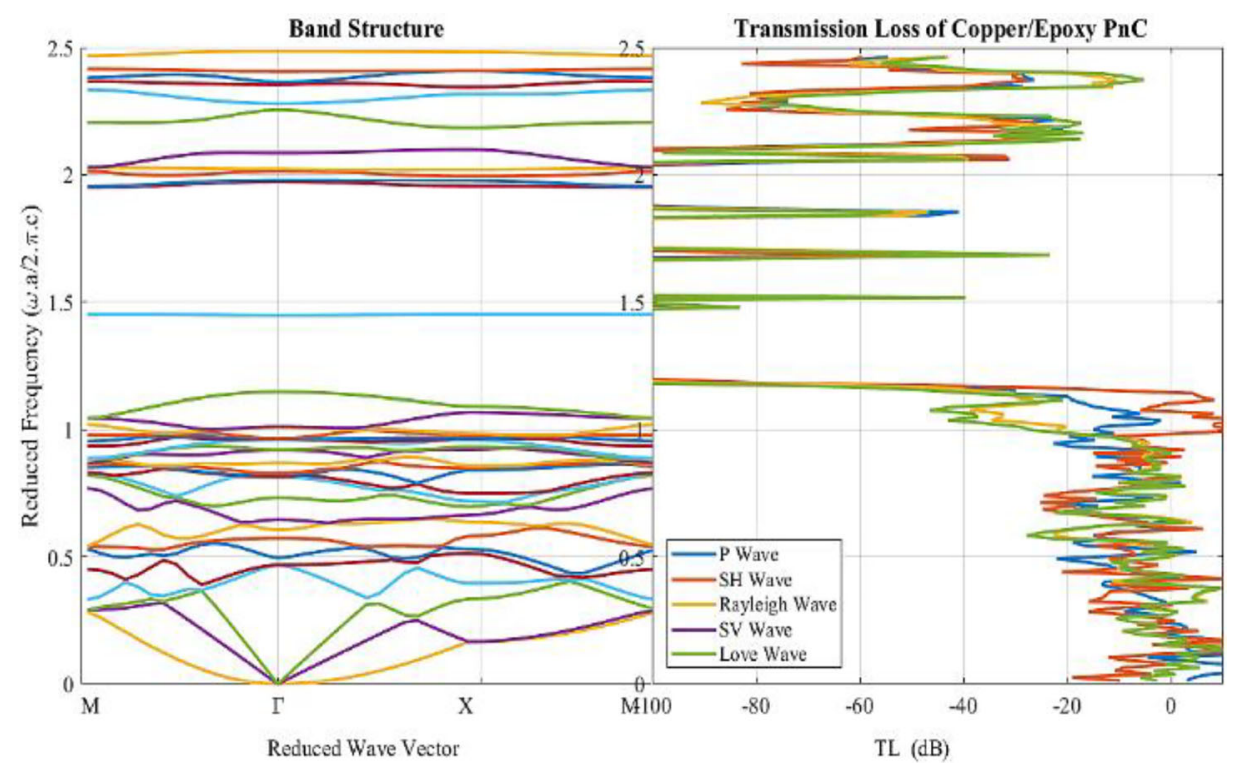

Figure 4. Band structure and transmission of Archimedean-like tiling in Grünbaum and Shephard notation $\left(3^{2}, 4,3,4\right)$ with cylindrical copper inclusion.

cylinders ( $\mathrm{Al}, \mathrm{Cu}, \mathrm{Si}$, and $\mathrm{W}$ ) embedded in epoxy. The ratio of $r / a$ for square lattice is 0.18 and $r / a_{o}$ for honeycomb lattice is 0.14 , respectively. In Figures 4-6, the Archimedean-like structures are shown with their transmission losses spectra. For comparison, we also present corresponding results for the different lattice (ladybug and bathroom) PnCs with different inclusions $(\mathrm{Cu}, \mathrm{Al}, \mathrm{Si}$, and $\mathrm{W})$. The frequency scale is the same for all lattices as expressed in $\omega a / 2 \pi c_{s_{-} e p o x y}$. As can be seen in Figures $4-6$, all of the structures studied possess three complete widely phononic band gaps with the first complete band gap being centered at the normalized frequency close to 1.2. It is important to note that the frequencies are almost independent of the number atoms per cell. These frequencies may be related only to the distance between neighboring cylinders. 


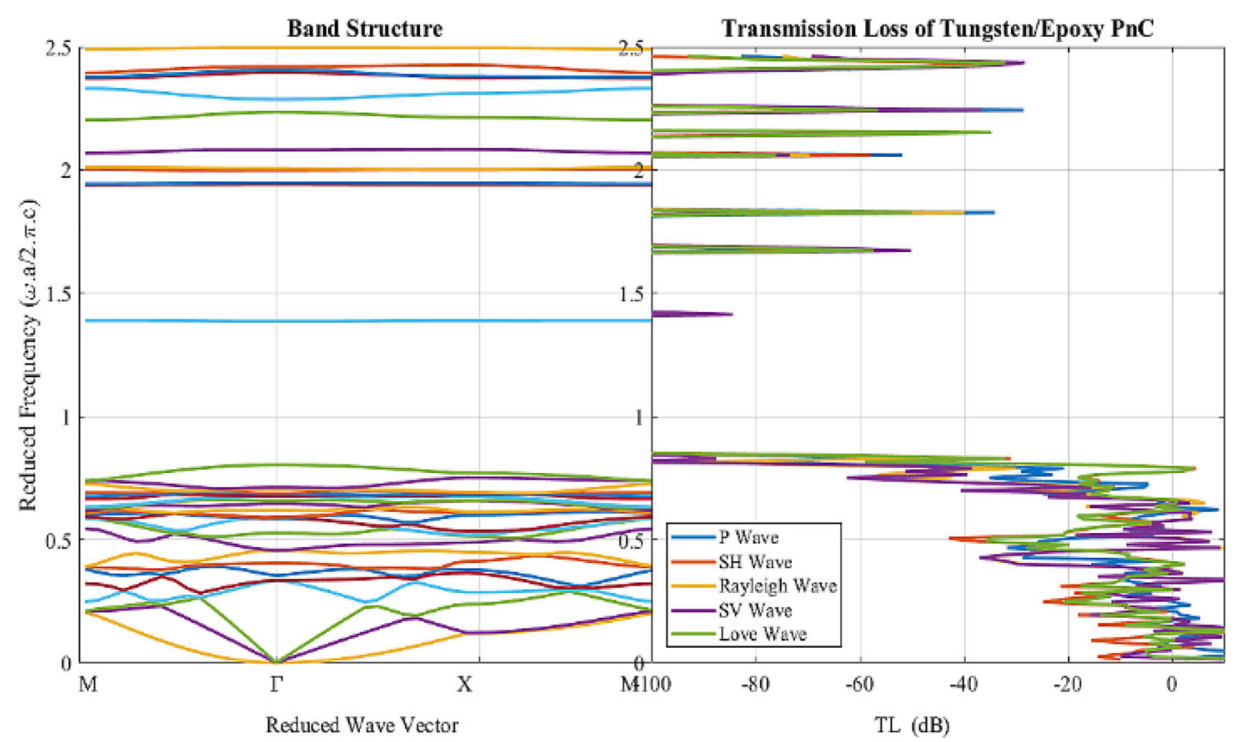

Figure 5. Band structure and transmission of Archimedean-like tiling in Grünbaum and Shephard notation $\left(3^{2}, 4,3,4\right)$ with cylindrical tungsten inclusion.

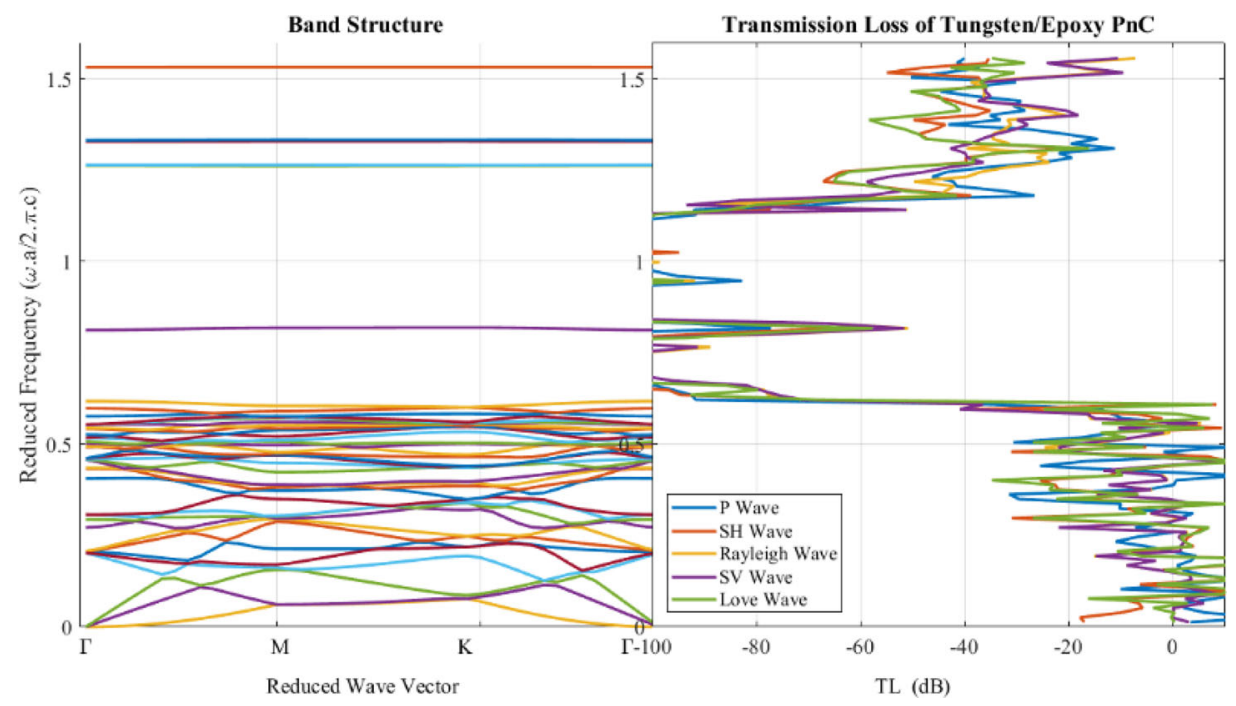

Figure 6. Band structure and transmission of Archimedean like tiling in Grünbaum and Shephard notation $(3,4,6,4)$ with cylindrical tungsten inclusion.

Especially, the positions and widths of the complete band-gaps were compared with the transmission loss spectra that were calculated for the investigated systems. Good agreement between band structures and transmission spectra calculations is observed.

Tables 1-5 show the main forbidden bands of PnCs. As seen in Table 1, the first band of the classical square lattice $\mathrm{PnC}$ with tungsten cylindrical inclusion is a $60 \%$ gap size with the center frequency 0.57 and the second band is a $35.85 \%$ gap size with the center frequency 1. 
Table 1. Full band-gap size variation classical square lattice PnC with cylindrical cross-section inclusion.

\begin{tabular}{|c|c|c|c|c|c|c|c|c|}
\hline \multirow[b]{2}{*}{ Bands } & \multicolumn{2}{|c|}{ Cop. } & \multicolumn{2}{|c|}{ Alum. } & \multicolumn{2}{|c|}{ Tung. } & \multicolumn{2}{|c|}{ Sil. } \\
\hline & $\begin{array}{c}\text { Mid } \\
\text { gap }(a / c)\end{array}$ & $\begin{array}{c}\text { Gap } \\
\text { size (\%) }\end{array}$ & $\begin{array}{c}\text { Mid } \\
\text { gap }(a / c)\end{array}$ & $\begin{array}{c}\text { Gap } \\
\text { size (\%) }\end{array}$ & $\begin{array}{c}\text { Mid } \\
\text { gap }(a / c)\end{array}$ & $\begin{array}{c}\text { Gap } \\
\text { size (\%) }\end{array}$ & $\begin{array}{c}\text { Mid } \\
\text { gap }(a / c)\end{array}$ & $\begin{array}{c}\text { Gap } \\
\text { size (\%) }\end{array}$ \\
\hline 1 & 0.62 & 17.61 & - & - & 0.57 & 60.96 & - & - \\
\hline 2 & 0.96 & 22.24 & 1.01 & 12.08 & 1.00 & 35.85 & 1.08 & 18.75 \\
\hline 3 & - & - & - & - & 1.53 & 0.09 & 1.55 & 1.13 \\
\hline
\end{tabular}

Table 2. Full band gap size variation of Archimedean-like tiling in Grünbaum and Shephard notation $\left(3^{2}, 4,3,4\right)$ PnC with cylindrical cross-section inclusion.

\begin{tabular}{|c|c|c|c|c|c|c|c|c|}
\hline \multirow[b]{2}{*}{ Bands } & \multicolumn{2}{|c|}{ Cop. } & \multicolumn{2}{|c|}{ Alum. } & \multicolumn{2}{|c|}{ Tung. } & \multicolumn{2}{|c|}{ Sil. } \\
\hline & $\begin{array}{c}\text { Mid } \\
\text { gap }(a / c)\end{array}$ & $\begin{array}{c}\text { Gap } \\
\text { size }(\%)\end{array}$ & $\begin{array}{c}\text { Mid } \\
\text { gap }(a / c)\end{array}$ & $\begin{array}{c}\text { Gap } \\
\text { size (\%) }\end{array}$ & $\begin{array}{c}\text { Mid } \\
\text { gap }(a / c)\end{array}$ & $\begin{array}{c}\text { Gap } \\
\text { size (\%) }\end{array}$ & $\begin{array}{c}\text { Mid } \\
\text { gap }(a / c)\end{array}$ & $\begin{array}{c}\text { Gap } \\
\text { size (\%) }\end{array}$ \\
\hline 1 & 1.30 & 23.11 & - & - & 1.10 & 53.05 & - & - \\
\hline 2 & 1.70 & 29.20 & 1.94 & 8.79 & 1.66 & 33.08 & 0.28 & 12.06 \\
\hline
\end{tabular}

Table 3. Full band-gap size variation of Archimedean-like tiling in Grünbaum and Shephard notation $\left(3^{2}, 4,3,4\right)$ PnC with square cross-section inclusion.

\begin{tabular}{|c|c|c|c|c|c|c|c|c|}
\hline \multirow[b]{2}{*}{ Bands } & \multicolumn{2}{|c|}{ Cop. } & \multicolumn{2}{|c|}{ Alum. } & \multicolumn{2}{|c|}{ Tung. } & \multicolumn{2}{|c|}{ Sil. } \\
\hline & $\begin{array}{c}\text { Mid } \\
\text { gap }(a / c)\end{array}$ & $\begin{array}{c}\text { Gap } \\
\text { size (\%) }\end{array}$ & $\begin{array}{c}\text { Mid } \\
\text { gap }(a / c)\end{array}$ & $\begin{array}{c}\text { Gap } \\
\text { size (\%) }\end{array}$ & $\begin{array}{c}\text { Mid } \\
\text { gap }(a / c)\end{array}$ & $\begin{array}{c}\text { Gap } \\
\text { size (\%) }\end{array}$ & $\begin{array}{c}\text { Mid } \\
\text { gap }(a / c)\end{array}$ & $\begin{array}{c}\text { Gap } \\
\text { size (\%) }\end{array}$ \\
\hline 1 & 1.41 & 19.39 & - & - & 1.21 & 48.79 & - & - \\
\hline 2 & 1.79 & 26.04 & 2.04 & 7.30 & 1.77 & 29.44 & 2.13 & 8.70 \\
\hline
\end{tabular}

Table 4. Full band-gap size variation of Archimedean-like tiling in Grünbaum and Shephard notation $\left(4,8^{2}\right) \mathrm{PnC}$ with cylindrical cross-section inclusion.

\begin{tabular}{|c|c|c|c|c|c|c|c|c|}
\hline \multirow[b]{2}{*}{ Bands } & \multicolumn{2}{|c|}{ Cop. } & \multicolumn{2}{|c|}{ Alum. } & \multicolumn{2}{|c|}{ Tung. } & \multicolumn{2}{|c|}{ Sil. } \\
\hline & $\begin{array}{c}\text { Mid } \\
\text { gap }(a / c)\end{array}$ & $\begin{array}{c}\text { Gap } \\
\text { size (\%) }\end{array}$ & $\begin{array}{c}\text { Mid } \\
\text { gap }(a / c)\end{array}$ & $\begin{array}{c}\text { Gap } \\
\text { size (\%) }\end{array}$ & $\begin{array}{c}\text { Mid } \\
\text { gap }(a / c)\end{array}$ & $\begin{array}{c}\text { Gap } \\
\text { size (\%) }\end{array}$ & $\begin{array}{c}\text { Mid } \\
\text { gap }(a / c)\end{array}$ & $\begin{array}{c}\text { Gap } \\
\text { size (\%) }\end{array}$ \\
\hline 1 & 1.35 & 4.72 & - & - & 1.15 & 36.15 & - & - \\
\hline 2 & 1.59 & 6.79 & - & - & 1.56 & 10.14 & - & - \\
\hline 3 & 1.78 & 8.02 & - & - & 1.76 & 11.60 & - & - \\
\hline
\end{tabular}

The first band of Archimedean-like tiling in Grünbaum and Shephard notation $\left(3^{2}, 4,3,4\right)$ PnC with tungsten cylindrical inclusion is a $53 \%$ gap size with the center frequency 1.10 and the second band is a $33.08 \%$ gap size with the center frequency 1.66 , as shown in Table 2.

The first band of Archimedean-like tiling in Grünbaum and Shephard notation $\left(3^{2}, 4,3,4\right)$ PnC with tungsten square inclusion is a $48.79 \%$ gap size with the center frequency 1.21 and the second band is a $29.44 \%$ gap size with the center frequency 1.77 as shown in Table 3.

The first band of Archimedean-like tiling in Grünbaum and Shephard notation $\left(4,8^{2}\right)$ PnC with tungsten cylindrical inclusion is a $36.15 \%$ gap size with the center frequency 1.15, as shown in Table 4. 
Table 5. Full band-gap size variation of Archimedean-like tiling in Grünbaum and Shephard notation $(3,4,6,4) \mathrm{PnC}$ with cylindrical cross-section inclusion.

\begin{tabular}{|c|c|c|c|c|c|c|c|c|}
\hline \multirow[b]{2}{*}{ Bands } & \multicolumn{2}{|c|}{ Cop. } & \multicolumn{2}{|c|}{ Alum. } & \multicolumn{2}{|c|}{ Tung. } & \multicolumn{2}{|c|}{ Sil. } \\
\hline & $\begin{array}{c}\text { Mid } \\
\text { gap }(a / c)\end{array}$ & $\begin{array}{c}\text { Gap } \\
\text { size (\%) }\end{array}$ & $\begin{array}{c}\text { Mid } \\
\text { gap }(a / c)\end{array}$ & $\begin{array}{c}\text { Gap } \\
\text { size (\%) }\end{array}$ & $\begin{array}{c}\text { Mid } \\
\text { gap }(a / c)\end{array}$ & $\begin{array}{c}\text { Gap } \\
\text { size (\%) }\end{array}$ & $\begin{array}{c}\text { Mid } \\
\text { gap }(a / c)\end{array}$ & $\begin{array}{c}\text { Gap } \\
\text { size (\%) }\end{array}$ \\
\hline 1 & 0.87 & 0.19 & - & - & 0.71 & 27.07 & - & - \\
\hline 2 & 1.08 & 34.79 & - & - & 1.04 & 42.55 & - & - \\
\hline
\end{tabular}

The first band of Archimedean-like tiling in Grünbaum and Shephard notation $(3,4,6,4)$ PnC with tungsten cylindrical inclusion is a $27.07 \%$ gap size with the center frequency 0.71 and the second band is a $42.55 \%$ gap size with the center frequency 1.04 as shown in Table 5.

\section{Conclusion}

In this study, the band structure and transmission properties of different patterned square lattice and honeycomb lattice PnCs with different cross-sections scatterers in the epoxy matrix were investigated based on FEM simulation. We can summarize the results that were described hereinabove:

- Archimedean-like tiling PnCs have an advantage on the location and number of the forbidden bands.

- $(3,4,6,4)$ it is remarkable that the honeycomb lattice has a wide band structure.

- As seen in Tables 2 and 3, in the same lattice geometry, the shape of the crosssection of the inclusions did not significantly affect the band structure.

- Although the classical square lattice PnC has a wide band, the Archimedean-like tiling PnCs have more band gaps at higher frequencies, and especially Grünbaum and Shephard notation $\left(3^{2}, 4,3,4\right) \mathrm{PnC}$ is remarkable with its broadband structure at high frequencies both cylindrical and square cross-section inclusions.

- The Archimedean-like tiling in Grünbaum and Shephard notation $(3,4,6,4)$ PnCs have band gaps at lower frequencies.

PnCs have led to the emergence of new research fields in the wave guide, filter, medical ultrasound, and RF communications fields.

\section{References}

1. R. H. Olsson and I. El-Kady, Microfabricated phononic crystal devices and applications, Meas. Sci. Technol. 20 (1), 012002 (2009). DOI: 10.1088/0957-0233/20/1/012002.

2. F. Ciampa, A. Mankar, and A. Marini, Phononic crystal waveguide transducers for nonlinear elastic wave sensing, Sci. Rep. 7 (1), 14712 (2017). DOI: 10.1038/s41598-017-14594-4.

3. S. Mohammadi et al., High-Q micromechanical resonators in a two-dimensional phononic crystal slab, Appl. Phys. Lett. 94 (5), 051906 (2009). DOI: 10.1063/1.3078284.

4. P. Zhang, and A. C. To, Broadband wave filtering of bioinspired hierarchical phononic crystal, Appl. Phys. Lett. 102 (12), 121910 (2013). https://doi.org/10.1063/1.3078284. DOI: $10.1063 / 1.4799171$. 
5. K. Lee et al., One-dimensional broadband phononic crystal filter with unit cells made of two nonuniform impedance-mirrored elements, AIP Adv. 3 (2), 022105 (2013). DOI: 10. 1063/1.4790638.

6. R. Wilson et al., Phononic crystal structures for acoustically driven microfluidic manipulations, Lab Chip 11, 323 (2011). DOI: 10.1039/c0lc00234h.

7. J. Li, Y. S. Wang, and C. Zhang, Finite element method for analysis of band structures of phononic crystal slabs with Archimedean-like Tilings, IEEE International Ultrasonics Symposium Proceedings, 2009. 1548-1551. DOI: 10.1109/ULTSYM.2009.5442087.

8. K. Ueda, T. Dotera, and T. Gemma, Photonic band structure calculations of two-dimensional Archimedean tiling patterns, Phys. Rev. B. 75 (19), 195122 (2007). DOI: 10.1103/ PhysRevB.75.195122.

9. Jovanović, R. Gajić, and K. Hingerl, Refraction and band isotropy in 2D square-like Archimedean photonic crystal lattices, Opt. Express 16, 4048 (2008). DOI: 10.1364/oe.16. 004048.

10. L. W. Schmerr, Jr., Fundamentals of Ultrasonic Nondestructive Evaluation a Modeling Approach (Springer International Publishing, Switzerland, 2016).

11. http://www.shenitech.com/support/support_soundspeed.htm (accessed Mar. 17, 2019) 